\title{
A randomized, double-blind, placebo-controlled trial to assess safety and tolerability during treatment of type 2 diabetes with usual diabetes therapy and either Cycloset $^{\mathrm{TM}}$ or placebo Richard E Scranton*1, J Michael Gaziano², Dean Rutty ${ }^{3}$, Michael Ezrokhi ${ }^{1}$ and Anthony Cincotta ${ }^{1}$
}

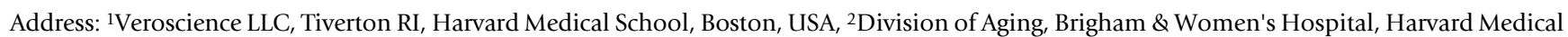
School, Boston, USA and ${ }^{3}$ Everest Clinical Research Services Inc., Toronto, Canada

Email: Richard E Scranton* - richard_scranton@veroscience.com; J Michael Gaziano - jmgaziano@partners.org; Dean Rutty - dean_rutty@ecrscorp.com; Michael Ezrokhi - michael_ezrokhi@veroscience.com; Anthony Cincotta - anthony_cincotta@veroscience.com

* Corresponding author

Published: 25 June 2007

BMC Endocrine Disorders 2007, 7:3 doi:10.1186/1472-6823-7-3

This article is available from: http://www.biomedcentral.com/1472-6823/7/3

(c) 2007 Scranton et al; licensee BioMed Central Ltd.

This is an Open Access article distributed under the terms of the Creative Commons Attribution License (http://creativecommons.org/licenses/by/2.0), which permits unrestricted use, distribution, and reproduction in any medium, provided the original work is properly cited.

\begin{abstract}
Background: Cycloset ${ }^{\mathrm{TM}}$ is a quick-release formulation of bromocriptine mesylate, a dopamine agonist, which in animal models of insulin resistance and type 2 diabetes acts centrally to reduce resistance to insulin- mediated suppression of hepatic glucose output and tissue glucose disposal. In such animals, bromocriptine also reduces hepatic triglyceride synthesis and free fatty acid mobilization, manifesting decreases in both plasma triglycerides and free fatty acids. In clinical trials, morning administration of Cycloset ${ }^{\mathrm{TM}}$ either as monotherapy or adjunctive therapy to sulfonylurea or insulin reduces $\mathrm{HbA}$ Ic levels relative to placebo by $0.55-\mathrm{I}$.2. Cycloset ${ }^{\mathrm{TM}}$ therapy also reduces plasma triglycerides and free fatty acid by approximately $25 \%$ and $20 \%$, respectively, among those also receiving sulfonylurea therapies. The effects of oncedaily morning Cycloset ${ }^{\mathrm{TM}}$ therapy on glycemic control and plasma lipids are demonstrable throughout the diurnal portion of the day (7 a.m. to 7 p.m.) across postprandial time points.
\end{abstract}

Methods/Design: 3,095 individuals were randomized in a 2:I ratio into a one year trial aimed to assess the safety and efficacy of Cycloset ${ }^{\mathrm{TM}}$ compared to placebo among individuals receiving a variety of treatments for type 2 diabetes. Eligibility criteria for this randomized placebo controlled trial included: age $30-80, \mathrm{HbAIc} \leq 10 \%$, diabetes therapeutic regimen consisting of diet or no more than two hypoglycemic agents or insulin with or without one additional oral agent (usual diabetes therapy; UDT). The primary safety endpoint will test the hypothesis that the rate of all-cause serious adverse events after one year of usual diabetes therapy (UDT) plus Cycloset ${ }^{\mathrm{TM}}$ is not greater than that for UDT plus placebo by more than an acceptable margin defined as a hazard ratio of 1.5 with a secondary endpoint analysis of the difference in the rate of serious cardiovascular events, (myocardial infarction, stroke, coronary revascularization or hospitalization for or angina or congestive heart failure). Efficacy analyses will evaluate effects of Cycloset ${ }^{\mathrm{TM}}$ versus placebo on change from baseline in $\mathrm{HbAlc}$, fasting glucose, body weight, waist circumference, blood pressure and plasma lipids.

Discussion: This study will extend the current data on Cycloset ${ }^{\mathrm{TM}}$ safety, tolerability and efficacy in individuals with type 2 diabetes to include its effects in combination with thiazolodinediones, insulin secretagogues, metformin, alphaglucosidase inhibitors and exogenous insulin regimens.

Trial registration: clinical trials.gov NCT00377676 


\section{Background}

Reduced insulin-mediated glucose disposal and impaired suppression of endogenous (hepatic) glucose production are dominant metabolic features of the obese state. [1] It has been estimated that $60-80$ percent of type 2 diabetic patients are obese. [2] Among representative species of all the major vertebrate classes of animals, the development of obesity and its accompanying insulin resistance has certain adaptive advantages. This adaptation improves survival in times of seasonal famine.[3] Many vertebrate species develop obesity and insulin resistance in preparation for hibernation, migration or during over-wintering periods when food availability is extremely low.[4] Extensive experimental evidence indicates that circadian neuroendocrine rhythms play a pivotal role in the development of seasonal changes in body fat stores and insulin sensitivity. Specifically, temporal changes in the interaction of two distinct circadian neural circadian oscillations, mediated in part by dopaminergic and serotonergic neurotransmitter activity, have been shown to regulate the dramatic seasonal alterations in body weight and body composition that are characteristic of all vertebrate classes from teleosts to mammals.[3] Additional data obtained in pigs and rats suggest that similar mechanisms may play a role in the development of nonseasonal (e.g., aging-related) obesity and insulin resistance. $[5,6]$

Bromocriptine mesylate, an ergot derivative, is a sympatholytic dopamine D2 receptor agonist that can exert inhibitory effects on serotonin turnover in the central nervous system.[7] Available evidence suggests that bromocriptine can reverse many of the metabolic alterations associated with insulin resistance and obesity by resetting central (hypothalamic) circadian organization of monoamine neuronal activities.[8] Additionally, dopamine agonist treatment reduces ventromedial, arcuate and paraventricular hypothalamic drive for increased hepatic glucose production, lipid synthesis and mobilization, and insulin resistance. $[9,10]$ When administered systemically [11-13] or into the cerebral ventricle [14] during the early hours of the light cycle, bromocriptine prevents or reverses seasonal fattening, insulin resistance and hyperinsulinemia, as well as decreases endogenous (hepatic) glucose production in mammals.

Cycloset $^{\mathrm{TM}}$ is a new quick-release oral formulation of bromocriptine mesylate that has been employed in clinical studies to examine the effectiveness of timed bromocriptine administration to humans with metabolic disease including type 2 diabetes. Cycloset ${ }^{\mathrm{TM}}$ has been shown to reduce insulin resistance, glucose intolerance and hyperlipidemia during Phase II clinical trials. In three Phase III clinical trials, Cycloset ${ }^{\mathrm{TM}}$ significantly improved hyperglycemia among obese individuals with type 2 diabetes [15] Based on these study results, the FDA issued an approva- ble letter for Cycloset ${ }^{\mathrm{TM}}$ for the treatment of type 2 diabetes. Full approval is conditional, in part, on the completion of a large, placebo-controlled, randomized trial in persons with type 2 diabetes to evaluate fully drugrelated adverse events with a high degree of certainty. The standard preparation of bromocriptine, Parlodel ${ }^{\oplus}$, has been used for over 25 years in persons with Parkinson's disease, prolactinomas, acromegaly, infertility, and postpartum lactation, many of whom also had diabetes. However, there have been no prospective, randomized, placebo-controlled trials conducted which permit the estimation of incidence rate differences and ratios necessary for ruling out a clinically meaningful increased risk for adverse events in a population of persons with type 2 diabetes. This study will provide data that permits the estimation of serious adverse event incidence rate differences and ratios between Cycloset ${ }^{\mathrm{TM}}$ and placebo when these agents are added to usual diabetes therapy. In addition to the evaluation of safety parameters, we intend to evaluate pre-specified efficacy parameters among patient subgroups. Specifically we intend to assess changes in Hba1c among a subgroup of the study subjects that were not adequately controlled on metformin and sulfonylurea therapy. Clinical studies that combined Cycloset ${ }^{\mathrm{TM}}$ with metformin were not part of the original Cycloset ${ }^{\mathrm{TM}}$ clinical program because metformin was not commercially available in the United States at the time the studies were initiated.

\section{Methods/Design \\ Analysis of safety}

The primary objective of the study is to determine in subjects with type 2 diabetes receiving Usual Diabetes Therapy (UDT) and HbA1c $\leq 10 \%$ :

1. Whether add-on therapy with Cycloset ${ }^{\mathrm{TM}}$ results in allcause rates of serious adverse events that are not higher than that of placebo by a pre-defined margin.

2. Whether add-on therapy with Cycloset ${ }^{\mathrm{TM}}$ results in disease-specific rates of serious cardiovascular adverse events that are not higher than that of placebo by non-inferiority margin of 1.5.

While the primary purpose of this study is to establish the safety profile of Cycloset ${ }^{\mathrm{TM}}$ in type 2 diabetes, any potential positive cardiovascular benefits will be evaluated as well.

\section{Analysis of efficacy}

Hba1c changes from baseline to 24 weeks between Cycloset $^{\mathrm{TM}}$ and Placebo among subjects with a baseline Hba1c of $\geq 7.5 \%$ among the following subgroups:

1. Treated at baseline with any Oral hypoglycemic agent (OHA) including injectable insulin secretagogues 
a. Metformin plus or minus one OHA or injectable insulin secretagogue

b. Sulphonylurea plus or minus one OHA or injectable insulin secretagogue

2. Treated at baseline with Metformin and one sulphonylurea

\section{Study design and setting}

This is a double blind, multi-center, placebo-controlled, parallel group, non-inferiority study in subjects with type 2 diabetes, comparing usual diabetes therapy (UDT) plus Cycloset $^{\mathrm{TM}}$ to UDT plus placebo. Following a two-week lead-in period, 3,095 subjects were randomized in a 2:1 ratio to UDT plus Cycloset ${ }^{\mathrm{TM}}$ or UDT plus placebo across 73 clinical centers in the United States and Puerto Rico. Subjects were expected to remain on study treatment for 12 months, the first six weeks of which constitute a dose titration period.

\section{Ethical considerations}

Full ethical approval for this study was obtained. An external Data Monitoring and Safety Committee monitored the study progress.

\section{Study interventions}

The experimental condition is Cycloset ${ }^{\oplus}$ in addition to the subject's current diabetes therapeutic regimen and lifestyle regimen. The control condition is placebo subject's current diabetes therapeutic regimen and lifestyle regimen. The regimen must consist of either diet, oral hypoglycemic agents (no more than 2), or insulin (alone or with no more than 1 oral hypoglycemic agent). The usual diabetes agents could not change during the first three months of the study. However, the dosages of the oral agents or insulin were modified as deemed appropriate by the study site investigator to achieve target glycemic goals recommended by the American Diabetes Association [16] After three months, alterations in the diabetes regimen were allowed if deemed necessary by the study site investigator to adhere to the ADA guidelines for achieving glycemic control. Prior to switching oral antidiabetic medication to an alternative anti-diabetic medication, titration of the current oral anti-diabetic medication up to maximum tolerated dose was preferred and recommended to the study site investigators. However, the change in the regimen could not include additions that resulted in a final regimen that exceed two oral agents (not including study medication), or insulin, plus one oral agent (not including study medication).

\section{Identification of eligible patients}

Patients meeting the inclusion criteria were identified by research centers across the United States which included nineteen centers from the Veteran Affairs Healthcare System.

\section{Confirmation of serious adverse events}

Study site investigators elicited information regarding serious adverse events. The study safety officer reviewed all relevant safety data obtained from the study sites. Narratives were written from source documents. All serious adverse events (SAE) were verified for accuracy of coding for inclusion in the database. All SAEs were reviewed by an external adjudication committee consisting of three physicians (two cardiologists and one endocrinologist). Whenever possible, the medical records corresponding with the event were also reviewed.

\section{Determining eligibility for the study}

The principle investigator at each site reviewed the screening laboratories and medical history and assessed eligibility of screened study subjects (see table 1 inclusion and exclusion criteria).

\section{Patient follow-up procedures}

After screening, patients were randomized and titrated to the maximum tolerated dose of the study drug over a six week period. After titration, subjects were seen every 3 months until study end (week 52) or early termination. Subjects were contacted 30 days after completion of study drug to record any adverse events occurring after treatment completion. See table 2 for visit schedule and assessments.

\section{Patient outcome measures}

Safety outcomes

Primary - all cause serious adverse events

Secondary - Composite of cardiovascular outcomes (myocardial infarction, hospitalized angina or congestive heart failure, stroke, or coronary revascularization)

\section{Additional safety parameters}

All adverse events, withdrawal due to an adverse event, laboratory values, vital signs and ECG

\section{Other clinical measures}

- Fasting lipid profile (total, LDL, HDL cholesterol, triglycerides) and

- HbA1c and

- Fasting plasma glucose level and

- Body weight, waist circumference, blood pressure 
Table I: Inclusion/Exclusion Criteria

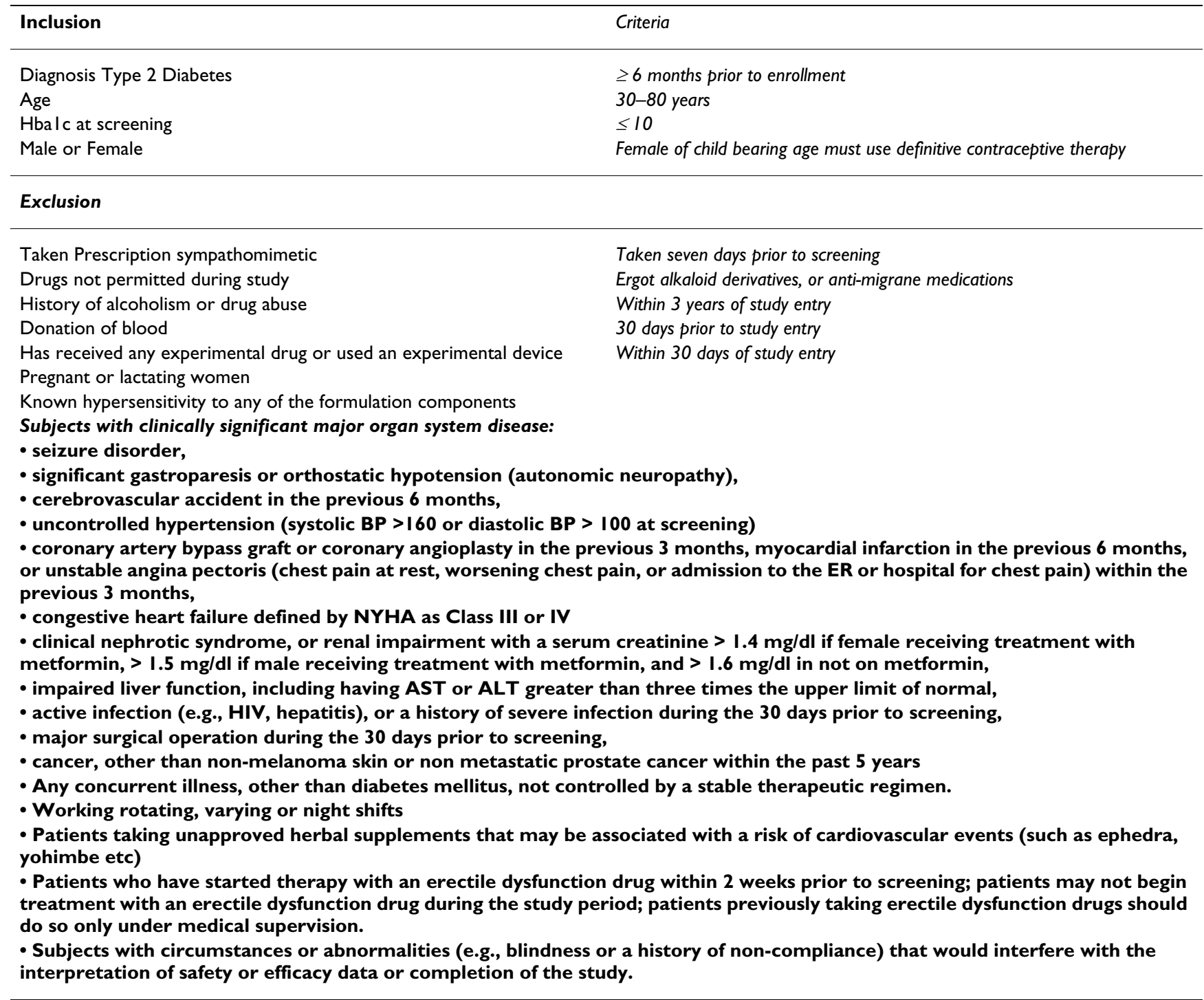

\section{Sample size considerations}

Sample size calculation for all-cause serious adverse events In order to test the primary hypothesis at a one-sided alpha $=.05$ and have a power of $1-\beta=0.90$ when the noninferiority margin is 1.5 , the total number of subjects with all-cause serious adverse events that must be observed during the study is 235 with a sample size of 2,991 (assuming the placebo rate of 0.08).

Power calculation for serious cardiovascular adverse events In order to test the hypothesis for serious cardiovascular adverse events as for the primary endpoint at a one-sided alpha $=.05$ when the non-inferiority margin is 1.5 , the final sample size of 3000 to 3300 subjects will provide at least $62 \%$ power, assuming a hypothetical rate of events of $3.43 \%$, or 103 to 113 cardiovascular serious adverse events.
Power calculation for efficacy analysis

With an expected $0.5 \%$ difference in mean change in HbA1c levels between the two treatment groups and a standard deviation of HbA1c level of $1.0 \%$ and the baseline and 24 week scores have a correlation of 0.50 then the effect size is $0.5 \% / 1.0 \%=0.50$. For the metformin/ sulphonylurea analysis, 160 subjects assuming a standard deviation of $1.0 \%$ will provide a power of $90 \%$ power to detect differences in mean changes of $0.5 \%$ or larger.

\section{Endpoints}

\section{Safety}

- The primary safety endpoint is the rate of time to first treatment emergent all-cause serious adverse events.

- Secondary safety endpoints include disease-specific rate of time to first treatment emergent serious cardiovascular 
Table 2: Study Flow Sheet

STUDY WEEK

\begin{tabular}{|c|c|c|c|c|c|c|c|c|c|c|c|c|c|c|c|}
\hline & & -2 & -1 & 0 & I & 2 & 3 & 4 & 5 & 6 & 12 & 24 & 36 & 52 & Termination $^{3}$ \\
\hline Assessment and Measurement & Pre-Screen & Screen & & ra & 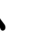 & & & & & & & & & & \\
\hline HbAIc: Local lab $<10$ & $*$ & $*$ & & & & & & & & & & & & & \\
\hline Clinic Visit & & $x$ & & $x$ & & & $x$ & & & $x$ & $x$ & $x$ & $x$ & $x$ & $x$ \\
\hline Medical History & & $x$ & & & & & & & & & & & & & \\
\hline Concomitant Meds and IIInesses & & $x$ & & & & & $x$ & & & $x$ & $x$ & $x$ & $x$ & $x$ & $x$ \\
\hline Pregnancy test* & & $x$ & & & & & & & & & & & & & \\
\hline Full Physical Exam, (Weight \& Waist Circumference)' & & $\mathrm{X}$ & & & & & & & & & & $x$ & & $\mathrm{x}$ & $\mathrm{x}$ \\
\hline Blood pressure & & $x x$ & & $x$ & & & $x$ & & & $x$ & $x$ & $X X$ & $\mathrm{x}$ & $x$ & $x$ \\
\hline Record Adverse events (AE) & & & & $x$ & & & $x$ & & & $x$ & $x$ & $x$ & $x$ & $x$ & $x$ \\
\hline ECG & & $x$ & & & & & & & & & & $x$ & & $x$ & $x$ \\
\hline Telephone Call to Subject for AE's Reports & & & & & $x$ & $x$ & & $\mathrm{x}$ & $x$ & & & & & & \\
\hline CBC \& Plasma Sample & & $x$ & & & & & & & & & & $x$ & & $x$ & $x$ \\
\hline Chemistry, LAE & & $\mathbf{X}^{\oplus}$ & & & & & & & & $x$ & & $x$ & & $x$ & $x$ \\
\hline $\mathrm{HbAlc}$ & & $x$ & & & & & & & & & $x$ & $x$ & $x$ & $x$ & $x$ \\
\hline Fasting Plasma Glucose ${ }^{2}$ & & $\mathrm{X}$ & & $x$ & & & $x$ & & & $x$ & $x$ & $x$ & $x$ & $x$ & $x$ \\
\hline Fasting Lipid Profile ${ }^{\star 2}$ & & $x$ & & & & & & & & & & $x$ & & $x$ & $x$ \\
\hline
\end{tabular}

\section{Notes on measures}

* Local lab documentation of $\mathrm{HbAl} \mathrm{c} \leq 10$ must be confirmed within 12 wks prior to conducting the Week-2 screening visit tests

- During Week-I any labs or tests unable to be performed at screening should be completed

* To include pregnancy test (beta-HCG) in women of childbearing potential.

- $\mathrm{CBC}$ with differential and platelets, urinalysis, liver function (ALT, AST, alkaline phosphatase and total bilirubin), BUN, creatinine, Na, K, Cl, bicarbonate, calcium, phosphorus, and albumin.

- Total cholesterol, triglycerides, HDL-cholesterol, and LDL-cholesterol.

I. for body mass index and waist circumference to be measured on subject in the supine position at the level of the umbilicus

2. for BMI Obtained in the morning after a minimum $8 \mathrm{~h}$ overnight fast

3. A full 52-week assessment must be performed whenever a subject terminates the study early.

adverse events (myocardial infarction, stroke, inpatient hospitalization for heart failure or angina, and revascularization surgery) and the individual specific rates.

\section{Efficacy}

Other clinical measures include the impact (either positive or negative) of Cycloset ${ }^{\mathrm{TM}}$ on HbA1c, fasting plasma glucose, weight, triglycerides lipids, blood pressure and patient tolerability after six months of therapy. Inasmuch as concurrent diabetes medications may change over the 52 week period with greater probability than at 24 weeks, the primary efficacy analyses will be based on changes from baseline to 24 weeks of treatment.

\section{Statistical analysis - safety and other clinical measures Safety}

The Intention-To-Treat (ITT) analysis set will include all subjects who were randomized to the study and received at least one dose of study treatment (either Cycloset ${ }^{\mathrm{TM}}$ or placebo) and will be used for evaluating all safety endpoints. The Evaluable Per-Protocol (EPP) analysis set will consist of those ITT subjects with no major protocol violations and with at least one post-randomization measurement of HbA1c, fasting plasma glucose, lipids, weight, waist circumference or blood pressure. The primary anal- ysis tested the hypothesis that the occurrence of all-cause serious adverse events for Usual Diabetes Therapy (UDT) plus Cycloset ${ }^{\mathrm{TM}}$ is not inferior to that for UDT plus placebo by a predefined margin. For this study, the predefined margin of non inferiority was defined as a hazard ratio (UDT plus Cycloset ${ }^{\oplus}$ versus UDT plus placebo) of 1.5. The hazard ratio and one-sided $96 \%$ confidence interval will be obtained from the Cox regression model with treatment and center effects. The non-inferiority will be supported by the result that the upper bound of the confidence interval is below 1.5. The proportional hazards assumption underlying the Cox model will be examined with appropriate alternative statistical models applied. Results based on the ITT analysis set will be considered primary and those based on EPP analysis supportive. The secondary safety endpoint is the time to the occurrence of first cardiovascular SAEs (myocardial infarction, stroke, in-patient hospitalization for heart failure, angina or revascularization surgery). These endpoints will be analyzed in the same manner as described above for the primary endpoint

\section{Other clinical measures}

Clinical efficacy analyses will also be conducted for subjects taking any oral hypoglycemic agent (OHA) or inject- 
able insulin secretagogue with a baseline HbA1c of $\geq$ $7.5 \%$. Additional subsets of this population include those receiving a) metformin with or without any OHA or injectable insulin secretagogue and b) sulphonylurea with or without any OHA or injectable insulin secretagogue. The difference in the change in the HbA1c from baseline to 24 weeks between Cycloset ${ }^{\mathrm{TM}}$ and placebo will be the primary efficacy measure. The analyses will be based on both a) ITT - last observation carry forward method and b) subjects with an HbA1c measurement at week 24 (completers). An analysis of covariance (ANCOVA) model will be used to test for a treatment difference while adjusting for the baseline hemoglobin $\mathrm{A}_{1 \mathrm{c}}$ value and center effect. Additional analyses will be used to assess the impacts from additional risk factors by including additional terms such as age, gender, body mass index at baseline, duration of disease history, and concomitant medications. Any significant effects from these potential risk factors will be further investigated. Analyses of treatment group differences in change from baseline in fasting plasma glucose, weight, waist circumference, triglycerides, lipid profile, and blood pressure will be explored within these defined groups.

We will also provide descriptive statistics on the changes in concomitant diabetes medications during the observational period (proportion of subjects adding therapy or increasing dose of preexisting therapy; proportion stopping therapy or decreasing dose; proportion not changing therapy). If warranted, additional analyses will assess the impact of such changes in concomitant diabetes medications during the observation period on difference between Cycloset $^{\mathrm{TM}}$ and placebo in change from baseline in HbA1c.

\section{Sub-study efficacy analyses}

Metformin/sulfonylurea efficacy analysis

The Intention-To-Treat efficacy analysis set (ITTe) will include all subjects who were randomized to the study and at the time of screening were treated with metformin plus a sulfonylurea and had a screening $\mathrm{HbA} 1 \mathrm{c}$ of $\geq 7.5 \%$, and received at least one dose of study treatment (either Cycloset $^{\mathrm{TM}}$ or placebo). The change in the HbA1c from baseline (screening visit week-2) to 24 weeks will be the primary efficacy measure. An analysis of covariance (ANCOVA) model will be used to test for a treatment difference while adjusting for the baseline hemoglobin $A_{1 c}$ value and center effect. For those patients who discontinued prior to 24 weeks, the last post-randomization observation will be carried forward. The Evaluable Per-Protocol efficacy analysis (EPPe) set will consist of those ITT subjects with no major protocol violations and with an Hba1c measurement at 24 weeks. Analyses of treatment group differences in change from baseline in fasting plasma glucose, weight, waist circumference, triglycerides, lipid profile, and blood pressure will be explored.
Prior to un-blinding the study data it is not possible to consider all possible adjustments needed to fully develop the appropriate model for determining changes in various efficacy parameters. We will further explore and deploy the appropriate methods of statistical analysis to fully discern the differences between Cycloset ${ }^{\mathrm{TM}}$ and placebo in this large study.

\section{Competing interests}

RS and AC are officers in the company Veroscience LLC which is developing Cycloset ${ }^{\mathrm{TM}}$ for the treatment of type 2 diabetes. ME is an employee of Veroscience. JG and DR report no competing interest involved with this trial.

\section{Authors' contributions}

RS is responsible for writing, technical review and assurance of accuracy of all protocol sections. AC is responsible for writing and technical review. JG and ME are responsible for technical review. DR is responsible for technical review of statistical section. All authors reviewed and approved the final protocol.

\section{References}

I. Ruderman N, Chisholm D, Pi-Sunyer X, Schneider S: The metabolically obese, normal-weight individual revisited. Diabetes I998, 47(5):699-7|3.

2. Cowie CC HMI: Physical and metabolic characteristics of persons with diabetes. Volume NIH publ. no. 95- / 468. Edited by: In Diabetes in America. National diabetes Data Group EBMD. National Institutes of Health; 1995: I 17-164.

3. Meier A CA: Circadian rhythms regulate the expression of the thrifty genotype/phenotype. diabetes $\operatorname{Rev} 1996,4: 464-487$.

4. Young R: Fat, energy and mammalian survival. Am Zool 1976, 16:699-710.

5. Cincotta AH, Schiller BC, Landry RJ, Herbert SJ, Miers WR, Meier $\mathrm{AH}$ : Circadian neuroendocrine role in age-related changes in body fat stores and insulin sensitivity of the male SpragueDawley rat. Chronobiol Int 1993, I 0(4):244-258.

6. Southern LL, Cincotta AH, Meier AH, Bidner TD, Watkins KL: Bromocriptine-induced reduction of body fat in pigs. J Anim Sci 1990, 68(4):931-936.

7. Erminio c P: Ergot compounds and brain function: neuroendocrine and neuropsychiatrics aspects. In Advances in Biochemical Psychopharmacology Edited by: Goldstein M LACDTM. New York , Raven; 1980:41-62.

8. Luo S, Luo J, Cincotta AH: Association of the antidiabetic effects of bromocriptine with a shift in the daily rhythm of monoamine metabolism within the suprachiasmatic nuclei of the Syrian hamster. Chronobiol Int 2000, I7(2): I55-I72.

9. Luo $\mathrm{S}$, Meier $\mathrm{AH}$, Cincotta $\mathrm{AH}$ : Bromocriptine reduces obesity, glucose intolerance and extracellular monoamine metabolite levels in the ventromedial hypothalamus of Syrian hamsters. Neuroendocrinology 1998, 68(I): 1- 10.

10. Bina KG, Cincotta $A H$ : Dopaminergic agonists normalize elevated hypothalamic neuropeptide $Y$ and corticotropinreleasing hormone, body weight gain, and hyperglycemia in ob/ob mice. Neuroendocrinology 2000, 7 I(I):68-78.

II. Cincotta AH, Schiller BC, Meier AH: Bromocriptine inhibits the seasonally occurring obesity, hyperinsulinemia, insulin resistance, and impaired glucose tolerance in the Syrian hamster, Mesocricetus auratus. Metabolism 1991, 40(6):639-644.

12. Cincotta $\mathrm{AH}$, Meier $\mathrm{AH}$, Southern LL: Bromocriptine alters hormone rhythms and lipid metabolism in swine. Ann Nutr Metab 1989, 33(6):305-314.

13. Cincotta $\mathrm{AH}$, MacEachern TA, Meier $\mathrm{AH}$ : Bromocriptine redirects metabolism and prevents seasonal onset of obese 
hyperinsulinemic state in Syrian hamsters. Am J Physiol 1993, 264(2 Pt I):E285-93.

14. Luo S, Liang $\mathrm{Y}$, Cincotta $\mathrm{AH}$ : Intracerebroventricular administration of bromocriptine ameliorates the insulin-resistant/ glucose-intolerant state in hamsters. Neuroendocrinology 1999 , 69(3): $160-166$.

15. Cincotta $\mathrm{AH}$, Meier $\mathrm{AH}$, Cincotta Jr M: Bromocriptine improves glycaemic control and serum lipid profile in obese Type 2 diabetic subjects: a new approach in the treatment of diabetes. Expert Opin Investig Drugs 1999, 8(10):1683-1707.

16. Standards of medical care in diabetes. Diabetes Care 2004, 27 Suppl I:SI5-35

\section{Pre-publication history}

The pre-publication history for this paper can be accessed here:

http://www.biomedcentral.com/1472-6823/7/3/prepub

Publish with Bio Med Central and every scientist can read your work free of charge

"BioMed Central will be the most significant development for disseminating the results of biomedical research in our lifetime. "

Sir Paul Nurse, Cancer Research UK

Your research papers will be:

- available free of charge to the entire biomedical community

- peer reviewed and published immediately upon acceptance

- cited in PubMed and archived on PubMed Central

- yours - you keep the copyright

Submit your manuscript here:

http://www.biomedcentral.com/info/publishing_adv.asp 